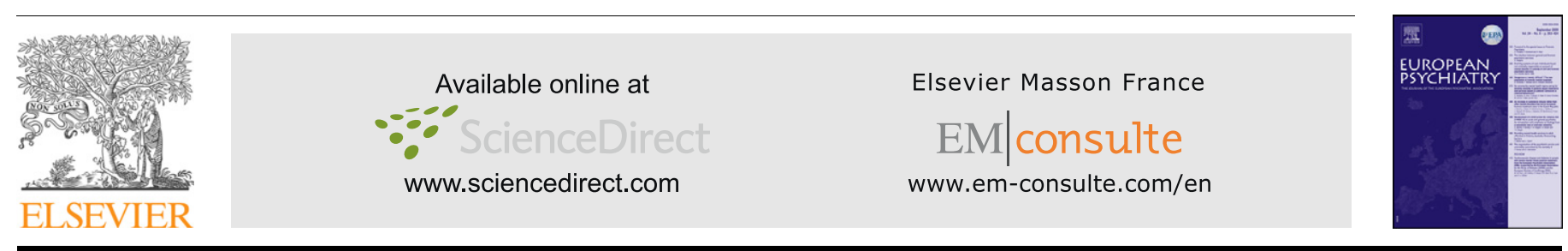

Letter to the editor

\section{Phenomenology of the social self in the prodrome of psychosis: From perceived negative attitude of others to heightened interpersonal sensitivity}

Qualitative changes in the experience of self in relation to others (i.e. the social self) have been consistently reported to be a feature of the "silent" initial part of the prodrome [4] and vulnerability to schizophrenia [2,8]. For example, increased interpersonal sensitivity, or changes in the felt naturalness of social interaction, often precedes behavioural manifestations such as social withdrawal and attenuated negative symptoms [3]. Thus, early experiential disturbances of social interaction might be an important early indicator of vulnerability to psychosis in helpseeking subjects.

The recent report by Salokangas et al. [6] offers crucial empirical evidence that seems to support this idea. Specifically, it shows that a not-yet psychotic, paranoia-tinged social sensitivity, consisting of the subjective experience of negative attitude of others (NAO) towards oneself, predicts prospective psychotic episodes (over a follow-up period of 5 years).

The study is a longitudinal hypothesis-testing of previous crosssectional findings [7], which revealed an association between NAO and escalating levels of psychoticism: broad vulnerability to psychosis, defined by self-reported psychotic-like experiences, and high risk of psychosis, assessed according to international criteria for prodromal/at risk mental states (i.e. transient or attenuated psychotic symptoms; presence of cognitive-perceptual basic symptoms; putative genetic risk and recent functional decline).

The study holds substantial clinical and conceptual appeal for several reasons. First, it shows that, although not formally included among the risk-indicators of transition to psychosis, certain forms of interpersonal sensitivity are actually predictive of imminent risk of psychosis.

Second, it coheres with other empirical studies pointing to certain forms of disturbed intersubjective experience as a feature of schizophrenia-related psychosis [2,5]. This includes a cluster of basic symptoms indicative of "interpersonal vulnerability", such as reduced ability to maintain or initiate social contacts, disturbances of expressive-emotional responsiveness, increased emotional reactivity to routine social interactions and unstable feelings of self-reference [2].

Finally, in our view, the study points to the heuristic value of adopting a sociorelational perspective in the clinical assessment of at-risk mental states. Despite the fact that various social inadequacies are frequently observed in prepsychotic prodromal phases, our current descriptive-conceptual repertoire is relatively modest, particularly within the field of early detection. In this context, the closest criteriological notion is attenuated negative symptoms [3], recently re-emphasized within the context of "early and broadly defined psychosis risk mental states" [1].
However, even attenuated negative symptoms, such as initial interpersonal withdrawal or declining social competences, might be of limited clinical specificity if only mapped onto the mere behavioural level (e.g. they can easily be secondary to nonpsychotic psychopathology such as anxiety or depressive disorders). But the same manifestations, when considered within a phenomenological context-that is, in relation to subtle feelings of self-reference and an initial paranoid interpersonal sensitivitymight ultimately be more illuminative of impending psychotic risk. Nonetheless, besides the "soft" paranoid tainting of social experiences, other salient experiential changes in the social self are also worth more focused clinical attention. For example, in the early prodrome of schizophrenia, the experience of interpersonal relatedness is often profoundly changed-although not necessarily in a paranoid configuration. This phenomenological alteration may exhibit a number of characteristics: a felt sense of loss of spontaneity (i.e., naturalness) in the very way of relating to and communicating with others; social hypo-hedonia (i.e. reduced vital engagement, social drive, a loss of pleasure in interactions with peers); experienced alterations of intersubjective resonance (e.g. over-impressionability or fading of empathic feelings towards others); propensity to de-socializing and solipsistic modes of experience (e.g. in the sense of alienation from the naturalness of common sense, or extraordinary, quasi-grandiose feelings of superhuman capacities and exceptionality, or radical incommunicability and dehumanization) ([4] and [8] for clinical examples). In our view, this diversity of experiential phenomena calls for a more systematic clinical focus on the intersubjective manifestations of vulnerability to psychosis, particularly with respect to the phenomenological underpinnings of social interaction.

\section{Disclosure of interest}

The authors declare that they have no conflicts of interest concerning this article.

\section{References}

[1] Keshavan MS, Delisi LE, Seidman LJ. Early and broadly defined psychosis risk mental states. Schizophr Res 2011, in press.

[2] Klosterkotter J, Ebel H, Schultze-Lutter F, Steinmeyer EM. Diagnostic validity of basic symptoms. Eur Arch Psychiatry Clin Neurosci 1996;246(3):147-54.

[3] Lencz T, Smith CW, Auther A, Correll CU, Cornblatt B. Nonspecific and attenuated negative symptoms in patients at clinical high-risk for schizophrenia. Schizophr Res 2004;68(1):37-48.

[4] Moller P, Husby R. The initial prodrome in schizophrenia: searching for naturalistic core dimensions of experience and behavior. Schizophr Bull 2000; 26(1):217-32.

[5] Oshima K, Okimura T, Yukizane T, Yasumi K, Iwawaki A, Nishikawa T, et al. Reliability and diagnostic validity for schizophrenia of the Japanese version of the Bonn Scale for Assessment of Basic Symptoms (BSABS). J Med Dent Sci 2010;57(1):83-94.

[6] Salokangas RK, Patterson P, Heinimaa M, Svirskis T, From T, Vaskelainen L, et al. Perceived negative attitude of others predicts transition to psychosis in patients at risk of psychosis. Eur Psychiatry 2011, in press. 
[7] Salokangas RK, Heinimaa M, Svirskis T, Laine T, Huttunen J, Ristkari T, et al. Perceived negative attitude of others as an early sign of psychosis. Eur Psychiatry $2009 ; 24(4): 233-8$.

[8] Stanghellini G. Vulnerability to schizophrenia and lack of common sense. Schizophr Bull 2000;26(4):775-87.

A. Raballo ${ }^{\mathrm{a}, *, \mathrm{~b}, \mathrm{c}}, \mathrm{J}$. Krueger ${ }^{\mathrm{a}}$

${ }^{a}$ Center for Subjectivity Research, University of Copenhagen, Njalsgade 140-142, Building 25, 5th floor, 2300 Copenhagen, Denmark ${ }^{\mathrm{b}}$ Department of Psychiatry, Psychiatric Center Hvidovre, University of Copenhagen, Brøndby, Denmark
${ }^{c}$ Department of Mental Health, AUSL di Reggio Emilia, Reggio Emilia, Italy

${ }^{*}$ Corresponding author. Tel.: +45 35328686; fax: +45 35328681 E-mail address: anr@hum.ku.dk (A. Raballo).

Received: 4 March 2011 Accepted: 17 March 2011

Please cite this article in press as: Raballo A, Krueger J. Phenomenology of the social self in the prodrome of psychosis: From perceived negative attitude of others to heightened interpersonal sensitivity. European Psychiatry (2011), doi:10.1016/j.eurpsy.2011.03.003 